\section{Polymer-capped magnetite nanoparticles change the 2D structure of DPPC model membranes $\dagger$}

\author{
Cristina Stefaniu, * Gerald Brezesinski and Helmuth Möhwald
}

Received 2nd April 2012, Accepted 1st June 2012

DOI: $10.1039 / \mathrm{c} 2 \mathrm{sm} 25773 \mathrm{~d}$

\begin{abstract}
The interaction of biocompatible and stimuli-responsive $\mathrm{Fe}_{3} \mathrm{O}_{4}$ nanoparticles (NPs) with DPPC model membranes was studied at the air-water interface. The pure NPs, pure DPPC and mixed DPPC-NP Langmuir layers have been characterized in situ by compression-expansion isotherms, infrared reflection-absorption spectrometry (IRRAS), grazing incidence X-ray diffraction (GIXD), total reflection X-ray fluorescence (TRXF), Brewster angle microscopy, and by atomic force microscopy after transfer onto a solid support. When dispersed in aqueous solution, the NPs are able to penetrate the phospholipid monolayer and to occupy a certain part of the interface defined by their own surface activity. The study reveals a largely phase separated system which actually masks an important interplay between the two compounds. Detailed GIXD measurements prove that the NPs are able to change the DPPC monolayer structure in a highly cooperative way by inducing a tighter in-plane packing. As IRRAS additionally supports, the effective size of the phospholipid polar head group is reduced due to partial dehydration and reorientation. The most surprising observation is the rigidification of the DPPC-NPs composite in a large range of surface pressures. Above the critical pressure, the phase separated NPs are squeezed out but the ones interacting with the DPPC molecules stay in the layer up to much higher lateral pressures. The interactions between NPs and DPPC stabilize the NPs at the interface. Only at very high pressures, all NPs are squeezed out. The amount of NPs in the monolayer has been quantified by TRXF. The desorption process is kinetically hindered.
\end{abstract}

\section{Introduction}

A panoply of new iron oxide nanoparticles (NPs) has been designed and synthesized in the last few years as valuable medical nano-tools. Generally, their coating enables them to bind, adsorb, or carry compounds such as drugs, proteins, enzymes, or antibodies, ${ }^{1}$ which can be then directed to a special tissue or organ using an external magnetic field. ${ }^{2}$ Nevertheless, despite the important number of newly synthesized NPs and their multiple biomedical applications, the knowledge of their interaction with cells or model membranes is still scarce.

The present study was focused on $\mathrm{Fe}_{3} \mathrm{O}_{4}$ NPs capped with a biocompatible and stimuli-responsive copolymer: $\mathrm{MEO}_{2} \mathrm{MA}_{90^{-}}$ co-OEGMA ${ }_{10}$ (Fig. 1). The system was previously developed and proved to be very promising for the biomedical field, acting as magnetic resonance imaging contrast enhancer and cell manipulation agent. ${ }^{3}$

Due to their possible use in biomedicine, different questions have to be answered: how do these NP systems interact with

Max Planck Institute of Colloids and Interfaces, Science Park Potsdam-Golm, Am Muehlenberg 1, D-14476 Potsdam, Germany. E-mail: stefaniu@mpikg.mpg.de

$\dagger$ Electronic supplementary information (ESI) available: Materials, experimental procedures and GIXD tables. See DOI: $10.1039 / \mathrm{c} 2 \mathrm{sm} 25773 \mathrm{~d}$ a cellular membrane? Are the NPs able to adsorb to or to penetrate the cellular membranes? Can the NPs show a special interaction and what would be their influence on the membrane structure?

In the present physical-chemical study, we investigated the interaction of $\mathrm{Fe}_{3} \mathrm{O}_{4} @ \mathrm{MEO}_{2} \mathrm{MA}_{90}-\mathrm{co}$-OEGMA 10 NPs with monolayers of dipalmitoylphosphatidylcholine (DPPC). Lipid monolayers have been chosen due to their general acceptance as excellent versatile model systems, able to mimic the outer leaflet of a cellular membrane.

As a prerequisite of understanding the interaction between the NPs and the model membrane, we previously studied and reported the interfacial properties of the NPs at the air-water interface..$^{4-7}$ The data proved to be very valuable for understanding the interaction process described herein.

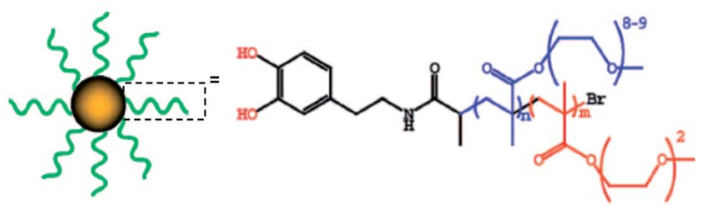

Fig. 1 Schematic representation of the $\mathrm{Fe}_{3} \mathrm{O}_{4} @ \mathrm{MEO}_{2} \mathrm{MA}_{90}-c o-$ OEGMA $_{10}$ NPs. 
Thus, surface pressure-area isotherms were combined with maximum insertion pressure (MIP) measurements, grazing incidence X-ray diffraction (GIXD), total reflection X-ray fluorescence (TRXF), infrared reflection-absorption spectrometry (IRRAS), atomic force microscopy (AFM) and Brewster angle microscopy experiments (BAM). The correlation of the data allows for the very first time a quantification of the influence of the NPs on the 2D phospholipid model membrane.

The study was conducted at $20{ }^{\circ} \mathrm{C}$, thus below the LCST (lower critical solution temperature) of the NPs in water $\left(43^{\circ} \mathrm{C}\right) .^{3}$

\section{Results and discussion}

\section{Insertion experiments}

Maximum insertion pressure experiments have been performed by injecting a defined amount of NPs underneath DPPC monolayers compressed to different initial lateral pressures. The surface area was kept constant and the increase in the surface pressure was monitored over time. In all experiments performed at starting pressures below $25 \mathrm{mN} \mathrm{m}^{-1}$, the NPs penetrate the lipid monolayer. The obtained equilibrium surface pressure of $\sim 25 \mathrm{mN} \mathrm{m}^{-1}$ is independent of the initial surface pressure
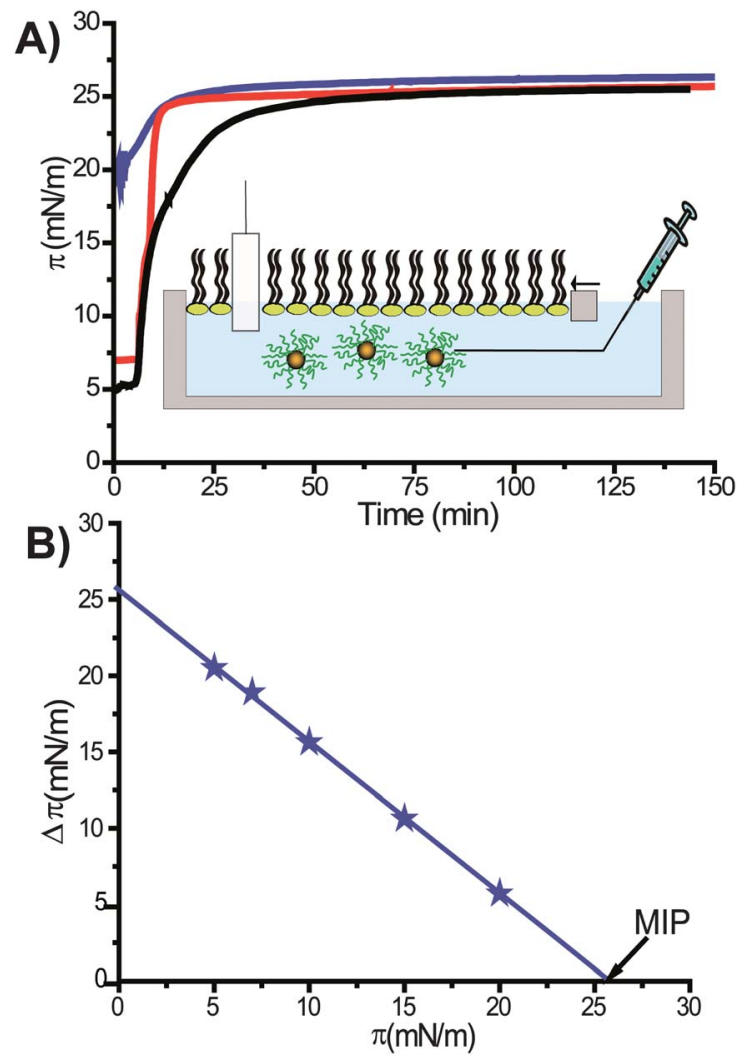

Fig. 2 (A) Variation of the surface pressure upon injection of a defined amount of $\mathrm{Fe}_{3} \mathrm{O}_{4} @ \mathrm{MEO}_{2} \mathrm{MA}_{90}-c o$-OEGMA ${ }_{10}$ NPs underneath DPPC monolayers compressed to different initial surface pressures (e.g.: 5 (black line), 7 (red line) and $20 \mathrm{mN} \mathrm{m}^{-1}$ (blue line)). For all experiments, the final concentration of the NPs in the subphase was $1.5 \times 10^{-3} \mathrm{mg}$ $\mathrm{ml}^{-1}$. The experiments were performed at $20^{\circ} \mathrm{C}$. (B) Determination of the maximum insertion pressure (MIP) of the NPs into the DPPC monolayer.
(Fig. 2A). This value corresponds to the equilibrium surface pressure of the NPs adsorbed at the bare air-water interface. ${ }^{6,7}$ Fig. 2B shows the increase in the surface pressure versus the initial surface pressure of the monolayer. The linear extrapolation gives a value of $25.5 \mathrm{mN} \mathrm{m}^{-1}$ for the maximum insertion pressure of the NPs into the DPPC model membrane.

No change in the surface pressure was observed when the NPs were injected underneath DPPC monolayers compressed to surface pressures higher than $25 \mathrm{mN} \mathrm{m}^{-1}$.

These findings demonstrate that the insertion process of the NPs into the DPPC monolayer is controlled by the surface activity of the NPs, and therefore, as previously reported, by the copolymer's surface activity. ${ }^{7}$ Since $\Delta \pi$ amounts also to $25.5 \mathrm{mN}$ $\mathrm{m}^{-1}$ for an uncompressed DPPC monolayer, the insertion process is obviously independent of the phase state of DPPC: liquid expanded or liquid condensed.

This observation is similar to the monolayer insertion ability of different poloxamers, which are known to effectively seal electrically damaged cell membranes. ${ }^{8-10}$ Similarly to our study, some poloxamers were reported as being able to insert into lipid films at a surface pressure below their maximal adsorption pressure, while larger polymers could insert only at pressures much lower than their respective maximal surface pressures. ${ }^{11}$

GIXD measurements have been performed in order to identify the influence of inserted NPs on the DPPC monolayer structure. Fig. 3B shows that after the adsorption of the NPs to a DPPC monolayer, initially compressed to $10 \mathrm{mN} \mathrm{m}^{-1}$, the tilt angle of

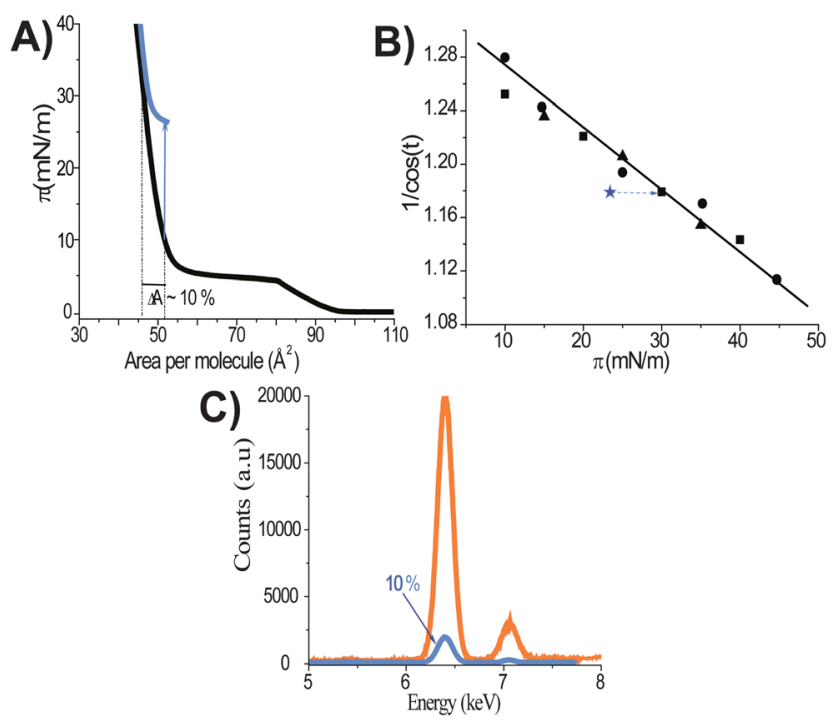

Fig. 3 (A) Compression isotherms of pure DPPC (black line) and of the mixed $\mathrm{Fe}_{3} \mathrm{O}_{4} @ \mathrm{MEO}_{2} \mathrm{MA}_{90}-c o$-OEGMA 10 NPs-DPPC (blue line) layers after NP adsorption. (B) Variation of the tilt angle with the surface pressure of a pure DPPC layer on different subphases (water - black triangles; PBS, pH 7.4 - black squares; Tris- $\mathrm{HCl}, \mathrm{pH} 8$ - black circles) compared to that of a mixed NPs-DPPC layer (blue star) obtained by adsorption of NPs from the subphase to a DPPC layer initially compressed to $10 \mathrm{mN} \mathrm{m}^{-1}$. The surface was kept constant during adsorption, and the GIXD measurement was performed after reaching the equilibrium at $25 \mathrm{~m} \mathrm{~m}^{-1}$. (C) X-ray fluorescence spectra showing the $\mathrm{Fe} \mathrm{K} \alpha$ and $\mathrm{Fe} \mathrm{K} \beta$ lines of a pure $\mathrm{Fe}_{3} \mathrm{O}_{4} @ \mathrm{MEO}_{2} \mathrm{MA}_{90}-c o$-OEGMA ${ }_{10} \mathrm{NP}$ layer (orange line) compared to that of a mixed DPPC-NPs layer (blue line) at the same lateral pressure of $25 \mathrm{mN} \mathrm{m}^{-1}$. 
the DPPC molecules is slightly reduced compared to that of a pure DPPC layer at the same lateral pressure $\left(25 \mathrm{mN} \mathrm{m}^{-1}\right)$. Such a tilt angle can be reached only at $\sim 31 \mathrm{mN} \mathrm{m}^{-1}$ in the pure DPPC layer. This indicates that the penetrated NPs do not only compress the lipid molecules like a piston, but influence clearly the monolayer structure. Since the mismatch between the area requirements of the DPPC head groups and the chains is responsible for the rather large tilt angle of the DPPC chains, the penetrated polymer chains obviously influence the head group orientation and/or hydration leading to a smaller tilt which has been found in pure DPPC monolayers only at higher surface pressures. This compression effect of the phospholipid monolayer is similar to the penetration mechanism of block copolymers like polystyrene - poly (ethylene oxide) ${ }^{12,13}$ or different poloxamers ${ }^{14}$ on DPPC monolayers.

Thus, the structural change of the DPPC ${ }^{15}$ layer consists of an increased in-plane packing density of the phospholipid molecules, which offers $\sim 10 \%$ of free interfacial space to the NPs (Fig. 3A). The NPs occupy this free surface until the maximum surface pressure is reached, as previously reported for their adsorption at the bare air-water interface. ${ }^{6,7}$ The fact that the NPs occupy $10 \%$ of the interface in the mixed monolayer was proved by in situ TRXF measurements. Thus, as depicted in Fig. $3 \mathrm{C}$, the intensity of the fluorescence $\mathrm{Fe} \mathrm{K} \alpha(6.4 \mathrm{keV})$ and $\mathrm{Fe}$ $\mathrm{K} \beta(7.06 \mathrm{keV})$ lines in the mixed DPPC-NPs layer is $\sim 10 \%$ of the intensity recorded from the pure NP monolayer at the same surface pressure $\left(25 \mathrm{mN} \mathrm{m}^{-1}\right.$, plateau region).

To summarize: the NPs are able to incorporate into a DPPC monolayer until they attain their characteristic maximum surface pressure. The free space offered to the NPs is limited by the in-plane packing density of the DPPC molecules, which is increased due to the interactions between DPPC and the polymer shell of the NPs.

\section{Co-spread mixtures}

Compression isotherms have been recorded for mixed Langmuir layers of $\mathrm{Fe}_{3} \mathrm{O}_{4} @ \mathrm{MEO}_{2} \mathrm{MA}_{90^{-}} \mathrm{co}-\mathrm{OEGMA}_{10} \mathrm{NPs}$ and DPPC (Fig. 4). Independent of the preparation method of the layers (DPPC spread on the surface of an aqueous dispersion of NPs, or

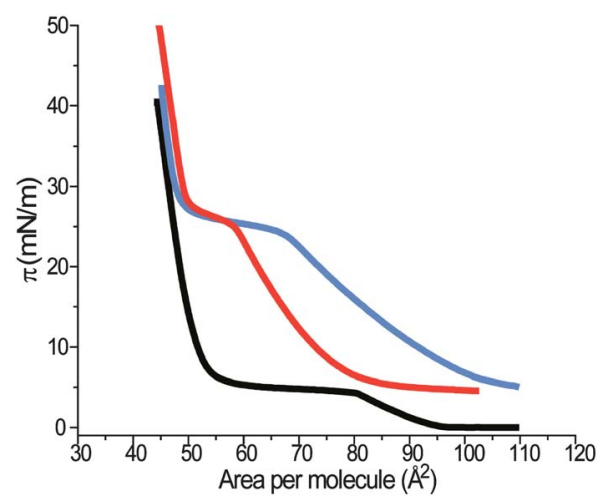

Fig. 4 Compression isotherms of pure DPPC spread on the surface of water (black line), and on a NP dispersion $\left(c=1.5 \times 10^{-3} \mathrm{mg} \mathrm{ml}^{-1}\right)$ (blue line) after waiting time of 15 minutes. Compression isotherm of mixed DPPC - $\mathrm{Fe}_{3} \mathrm{O}_{4} @ \mathrm{MEO}_{2} \mathrm{MA}_{90}$-co-OEGMA 10 NP layer co-spread on the surface of water (red line). The subphase temperature was $20^{\circ} \mathrm{C}$.
DPPC spread together with the NPs from a chloroform solution), the isotherms exhibit the same shape. Thus, the characteristic plateau region of the pure DPPC layer is present at $5 \mathrm{mN}$ $\mathrm{m}^{-1}$, indicating that the phospholipid phase transition pressure from the liquid expanded (LE) to the liquid condensed (LC) phase is not perturbed by the presence of the NPs in the monolayer. Due to the NPs' presence in the monolayer, this phase transition starts apparently at a larger area per DPPC molecule. Therefore, the entropy change connected with the LE/LC transition cannot be estimated. Additionally, a second plateau region appears at a surface pressure of $25 \mathrm{mN} \mathrm{m}^{-1}$. As previously reported, this plateau region marks the conformational changes of the copolymer chains from a less hydrated pancake-like conformation to a more hydrated brush-like conformation. ${ }^{4}$ The data indicate that the two components, DPPC and NPs, conserve their individual properties in the mixed layer.

Brewster angle microscopy experiments confirmed the phase separation between NPs and DPPC. Fig. 5 presents the pictures of mixed DPPC-NP layers compared to the pure DPPC layers at the same surface pressures. Thus, already at low surface pressures $\left(3 \mathrm{mN} \mathrm{m}^{-1}-\right.$ Fig. $\left.5 \mathrm{~B}\right)$, patches of DPPC in a LE state can be seen surrounded by NPs. Upon increasing the lateral pressure $\left(5 \mathrm{mN} \mathrm{m}^{-1}\right.$ - Fig. 5D) ordered LC domains of DPPC appear as bright spots in the segregated patches of liquid DPPC. However, the DPPC LC domains formed in two-phase coexistence region of the mixed layer do not express the multi-lobed shape characteristic for the enantiomerically pure DPPC ${ }^{16,17}$ (compare Fig. 5E and F, as well as $\mathrm{G}$ and $\mathrm{H}$ ). As previously reported for other systems, the change in the DPPC domain shape could be due to the presence of NPs which modify the line tension between the LC and LE phases compared to that of a pure lipid monolayer. ${ }^{18,19}$ This hints at (i) a partial miscibility of the polymer chains and DPPC and (ii) an enrichment of the polymer chains at the domain boundary. Thus, the line tension effect on the domain shape is stronger than the molecular chirality in our mixed system.

Moreover, the NPs separate patches of the now fully condensed DPPC even at high lateral pressure $\left(20 \mathrm{mN} \mathrm{m}^{-1}\right)$, while the pure DPPC layers show at this surface pressure a homogeneous condensed film (Fig. 5I and J).

The fast adsorption process of the NPs from the subphase to the DPPC monolayer prevented the investigation of mixed layers at surface pressures below $25 \mathrm{mN} \mathrm{m}^{-1}$. To overcome this problem, GIXD experiments have been performed on co-spread layers at the air-water interface. Additionally, to understand the influence of the pure copolymer on the DPPC structure, mixed co-spread layers of DPPC-polymer $\mathrm{MEO}_{2} \mathrm{MA}_{90^{-}} \mathrm{co}-\mathrm{OEGMA}_{10}$ have been studied.

Thus, the structure of the LC phase of DPPC was investigated by GIXD. The results show clearly the existence of only one liquid-crystalline phase, with a changed structure, compared to pure DPPC. BAM experiments showed an enrichment of the polymer chains at the domain boundary. However, since only one homogeneous monolayer structure is observed, it is obvious that the NPs changed the DPPC structure in a highly cooperative way. Fig. 6 indicates that in the surface pressure range between 10 and $30 \mathrm{mN} \mathrm{m}^{-1}$ the DPPC molecules in the mixed layers are characterized by a drastically smaller tilt angle (with respect to the normal to the interface) compared to the structure of the pure 


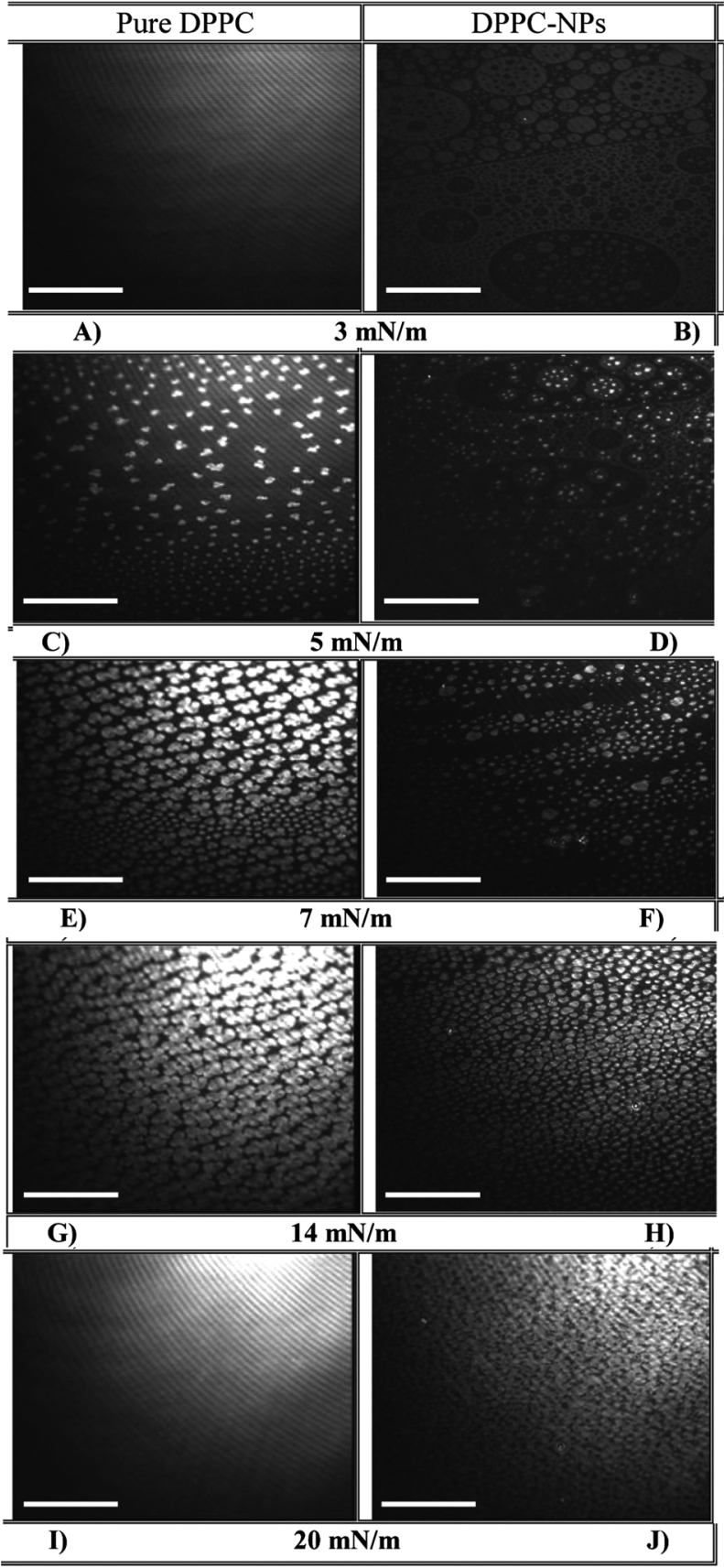

Fig. 5 Brewster angle microscopy images of mixed DPPC $-\mathrm{Fe}_{3} \mathrm{O}_{4} @$, $\mathrm{MEO}_{2} \mathrm{MA}_{90}-\mathrm{co}$-OEGMA 10 NP layers compared to BAM images of pure DPPC layers at different surface pressures (water subphase at $20^{\circ} \mathrm{C}$ ). Scale bar is $100 \mu \mathrm{m}$ for all images.

DPPC at the same pressure. The data have been plotted as $1 / \cos (t)$ versus $\pi$ since this can be described by a linear relationship. ${ }^{20}$ The tilt angle in the mixed system changes much less on compression than in the pure DPPC layer and corresponds, e.g., at $10 \mathrm{mN} \mathrm{m}^{-1}$, already to the one observed only at $30 \mathrm{mN}$ $\mathrm{m}^{-1}$ in the pure layer. This indicates that the presence of the NPs in the DPPC layer induces, even at low surface pressures, a very tight in-plane packing of the DPPC molecules which changes only slightly upon compression (rigidification effect).

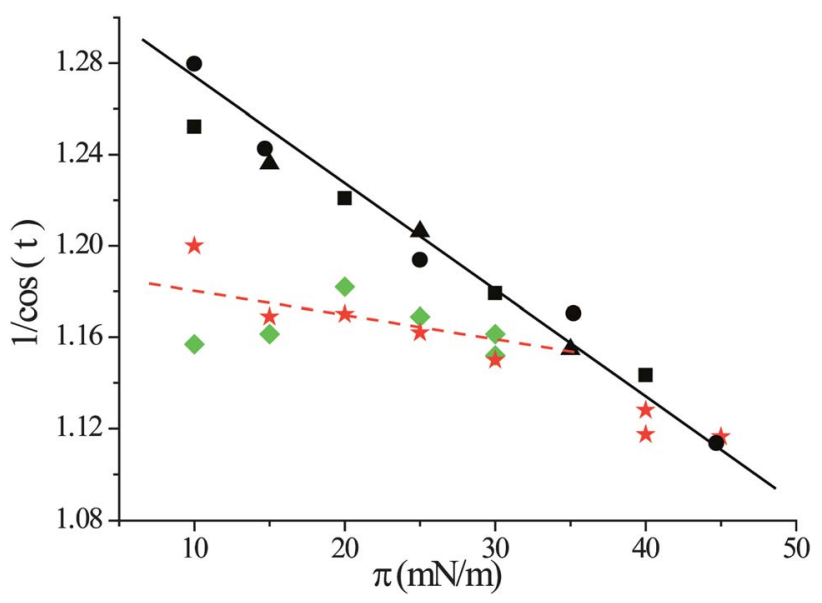

Fig. 6 Variation of the tilt angle with the surface pressure of a pure DPPC layer (water subphase - black triangles; PBS, pH 7.4 - black squares; Tris- $\mathrm{HCl}, \mathrm{pH} 8$ - black circles) compared to that of a mixed DPPC- $\mathrm{Fe}_{3} \mathrm{O}_{4} @ \mathrm{MEO}_{2} \mathrm{MA}_{90}-c o-\mathrm{OEGMA}{ }_{10} \mathrm{NP}$ layer (red stars) obtained by co-spreading and to that of a mixed DPPC- $\mathrm{MEO}_{2} \mathrm{MA}_{90^{-}}$-coOEGMA $_{10}$ layer (green diamonds) obtained by co-spreading on water subphase at $20^{\circ} \mathrm{C}$.

Furthermore, the almost overlapping of the GIXD data obtained for the DPPC-NP layers and DPPC-copolymer layer (at the same copolymer concentration) show clearly that the copolymer is dictating the changes produced in the DPPC monolayer structure (Fig. 6).

Despite the similar mechanism of interaction between the membrane sealers poloxamers ${ }^{14}$ and our NPs, to the best of our knowledge, this is the very first study reporting that polyethylene glycol co-polymers can have such a strong influence on the DPPC 2D structure.

The most unexpected result is that up to a lateral pressure of $\sim 35 \mathrm{mN} \mathrm{m}^{-1}$, which is higher than the MIP, the polymer capped NPs have a strong influence on the DPPC structure. The phase separated parts of the mixed DPPC-NPs layer react differently on the layer compression. The soft polymer part can be easily compressed to a higher density, whereas the rigid DPPC-NPs part containing some polymer chains is almost incompressible.

The GIXD results can be correlated with the compression isotherms to calculate the percentage of interface occupied by the NPs. Thus Fig. 6 allows determination of the lateral pressure at which the pure DPPC layer has the same structure as DPPC in the mixed layer. Changing the lateral pressure from $10 \mathrm{mN} \mathrm{m}^{-1}$ to $35 \mathrm{mN} \mathrm{m}^{-1}$ in the mixed layer corresponds to the change from 30 to $35 \mathrm{mN} \mathrm{m}^{-1}$ in the pure layer. ${ }^{15}$ Thus, by using the corresponding area values of the compression isotherm of pure DPPC and of the isotherm of the mixed DPPC-NPs layer, the percentage of area occupied by the NPs in the mixed layer has been calculated (Fig. 7B). Additionally, in situ TRXF results have been used to calculate this percentage independently. Thus, Fig. 7A is comparing the intensity of the $\mathrm{Fe} \mathrm{K} \alpha$ line of pure NP Langmuir layers (orange dots) with that of mixed DPPC-NP layers (blue stars). Fig. 7B shows that the values obtained by the two independent approaches are in a very good agreement.

The above presented data revealed the co-existence of NPs and DPPC molecules in mixed monolayers below $25 \mathrm{mN} \mathrm{m}^{-1}$ as well 


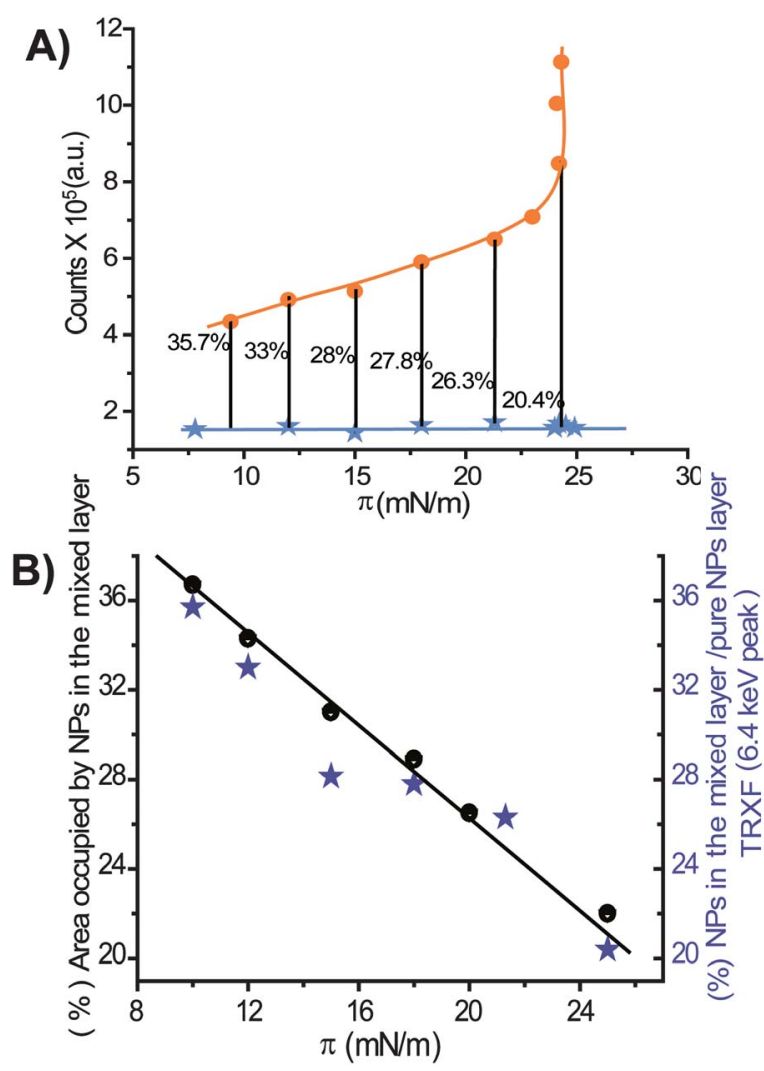

Fig. 7 (A) X-ray fluorescence intensity of the $\mathrm{Fe} \mathrm{K} \alpha(6.4 \mathrm{keV})$ line from a pure $\mathrm{Fe}_{3} \mathrm{O}_{4} @ \mathrm{MEO}_{2} \mathrm{MA}_{90^{-}}-\mathrm{o} o$-OEGMA ${ }_{10} \mathrm{NP}$ layer (orange dots) and a mixed DPPC-NPs layer (blue stars) used to calculate the percentage of the NPs in the mixed DPPC-NPs layer. (B) The correlation of the percentage of the area occupied by the NPs in the mixed layer (based on the GIXD data - black circles) with the percentage intensity from the $\mathrm{X}$-ray fluorescence measurements (blue stars) $-\mathrm{K} \alpha(6.4 \mathrm{keV})$ line.

as the influence of the NPs on the DPPC structure. In the following, the study will be focused on understanding the behavior of the mixed layers at and above this surface pressure. In this respect, the GIXD results indicate that above surface pressures of $\sim 35 \mathrm{mN} \mathrm{m}^{-1}$, the arrangement of DPPC molecules in the mixed DPPC-NP layers is characterized by the same monolayer structure as in pure DPPC layers (Fig. 6). Thus, the influence of the NPs on the DPPC molecules seems to be abolished at such high surface pressures.

Indeed, the compression-expansion isotherms of the mixed DPPC-NP layers (Fig. 8A) hint at the squeezing out of the NPs from the interface: First, above $30 \mathrm{mN} \mathrm{m}^{-1}$, the profile of the mixed layers is very similar to the one of the pure DPPC. The slope indicating the compressibility of the layer is almost identical even if the area is slightly larger in the mixed layer indicating that still some NPs are present in the layer, obviously stabilized by NP-lipid interactions. Second, an important hysteresis is recorded upon compression-expansion of the mixed layers as depicted in Fig. 8A. Also, the length of the plateau at $25 \mathrm{mN} \mathrm{m}^{-1}$ is reduced upon each additional compression step. As previously reported, on the plateau region $\left(25 \mathrm{mN} \mathrm{m}^{-1}\right)$ the NPs' copolymer shell changes the conformation from a less hydrated pancake-like conformation to the more hydrated brush-like structure. ${ }^{4}$
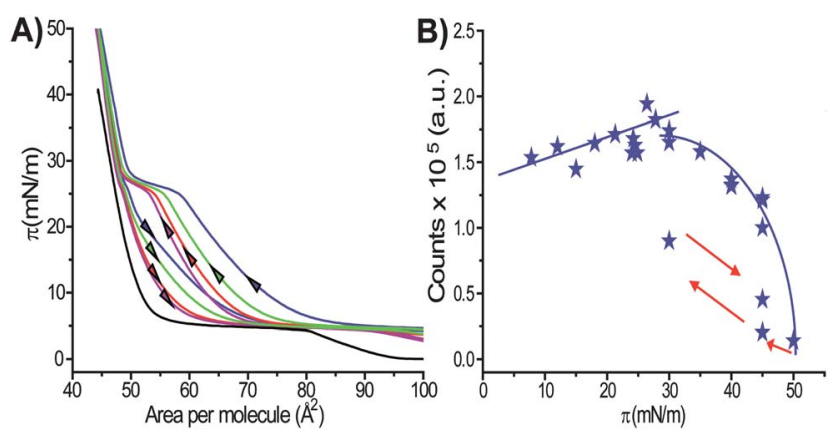

Fig. 8 (A) Compression isotherms of pure DPPC spread on water (black line), compared to the compression-expansion isotherms of mixed $\mathrm{Fe}_{3} \mathrm{O}_{4} @ \mathrm{MEO}_{2} \mathrm{MA}_{90}-c o$-OEGMA 10 NP-DPPC layers co-spread on water $\left(1^{\text {st }}\right.$ cycle - blue line; $2^{\text {nd }}$ cycle - green line; $3^{\text {rd }}$ cycle - red line; $4^{\text {th }}$ cycle - violet line). The subphase temperature was $20^{\circ} \mathrm{C}$. (B) Variation of the intensity of the fluorescence $\mathrm{Fe} \mathrm{K} \alpha(6.4 \mathrm{keV})$ line with compression/ expansion of the mixed DPPC-NPs layer.

Additionally, in the pure NP layers, due to the steric repulsion of the copolymer chains, the NP interfacial concentration is kept constant by desorbing the excess of NPs into the subphase. The NP desorption is a kinetically controlled process, which is much slower than the adsorption process. ${ }^{7}$ Considering the decrease of the length of the plateau region (Fig. 8A) an estimation can be made showing that upon the first compression-expansion cycle, $25 \%$ of the NPs are desorbed from the interface to the subphase, while after the $4^{\text {th }}$ cycle, the amount of squeezed out NPs is increased to $46 \%$ from the initial amount. The percentage could seem low but it has to be noted that upon expansion the NPs are re-adsorbed to the interface.

The answer to the question of the presence of the NPs in the mixed layer at high surface pressures was given by in situ total reflection X-ray fluorescence experiments. Thus, Fig. 8B presents the variation of the intensity of the fluorescence $\mathrm{Fe} \mathrm{K} \alpha(6.4 \mathrm{keV})$ line with the lateral pressure of the mixed DPPC-NP layer. Before discussing the squeezing out process of the NPs from the interface, a few remarks are needed. Thus, at the first glance it is surprising that over the whole range between 5 and $30 \mathrm{mN} \mathrm{m}^{-1}$ the measured fluorescence intensity is more or less constant or at least only slightly increasing. The expected tendency would be a continuous increase of the intensity until the maximum NP concentration is reached at $25 \mathrm{mN} \mathrm{m}^{-1}$. This apparent discrepancy could be simply explained by the fact that upon compression more and more space is occupied by the liquid condensed areas of DPPC (see Fig. 5). The phase separated NPs are compressed to a higher packing density but occupy less and less surface space. Thus, the increase of the NPs' density at the interface, which would lead to an increase in the fluorescence intensity, is compensated by their decreased allocated area.

As presented in Fig. 8B, the in situ TRXF experiments indicate desorption of the NPs from the DPPC layer at surface pressures above $35 \mathrm{mN} \mathrm{m}^{-1}$. Thus, at $40 \mathrm{mN} \mathrm{m}{ }^{-1}, 20 \%$ of the NPs are desorbed into the subphase. Moreover, a time dependent desorption process could be measured only above $40 \mathrm{mN} \mathrm{m}^{-1}$. Below this surface pressure, the mixed layers were highly stable, with no change in the measured fluorescence intensity of Fe over a period of 2 hours. The data indicate that at $45 \mathrm{mN} \mathrm{m}^{-1} 30 \%$ of 
the NPs are desorbed and this amount could be increased to $42 \%$ by keeping the surface pressure constant for $35 \mathrm{~min}$. At $50 \mathrm{mN} \mathrm{m} \mathrm{m}^{-1} 83 \%$ of the NPs have been desorbed into the subphase. This is in very good agreement with the $80 \%$ of desorbed NPs calculated by comparing the small remaining plateau obtained from the first expansion isotherm (Fig. 8A blue line). Thus, the data indicate that upon fast compression to high surface pressures, $20 \%$ of the initial amount of NPs are still remaining in the DPPC monolayer. This must be the amount of NPs which are strongly interacting with the DPPC head groups. Fig. $8 \mathrm{~B}$ confirms as well that upon expansion of the layer, the readsorption of the NPs to the interface occurs. A quantification of the desorption kinetics is, however, not possible due to the influence of too many factors. But at least qualitatively one can say that the desorption barrier is higher in the mixed layer compared to the pure NP layer due to interactions between the polymer chains and the DPPC head groups.

The NP desorption process could be monitored as well by the use of BAM and AFM. Thus, on the plateau region $\left(25-27 \mathrm{mN} \mathrm{m}^{-1}\right)$ both techniques revealed the coexistence of the two components. At these surface pressures, the highly concentrated regions of the segregated NPs are drawing different patterns into the mixed monolayers (Fig. 9). These phase separated domains of NPs are observed up to surface pressures of $45 \mathrm{~m} \mathrm{~N} \mathrm{~m}^{-1}$. At $50 \mathrm{mN} \mathrm{m}^{-1}$, the

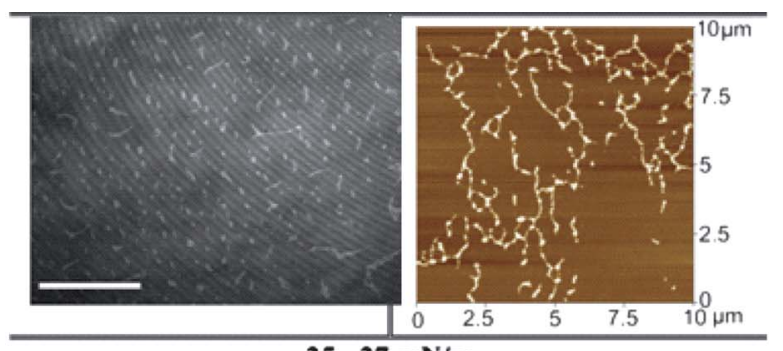

$25-27 \mathrm{mN} / \mathrm{m}$

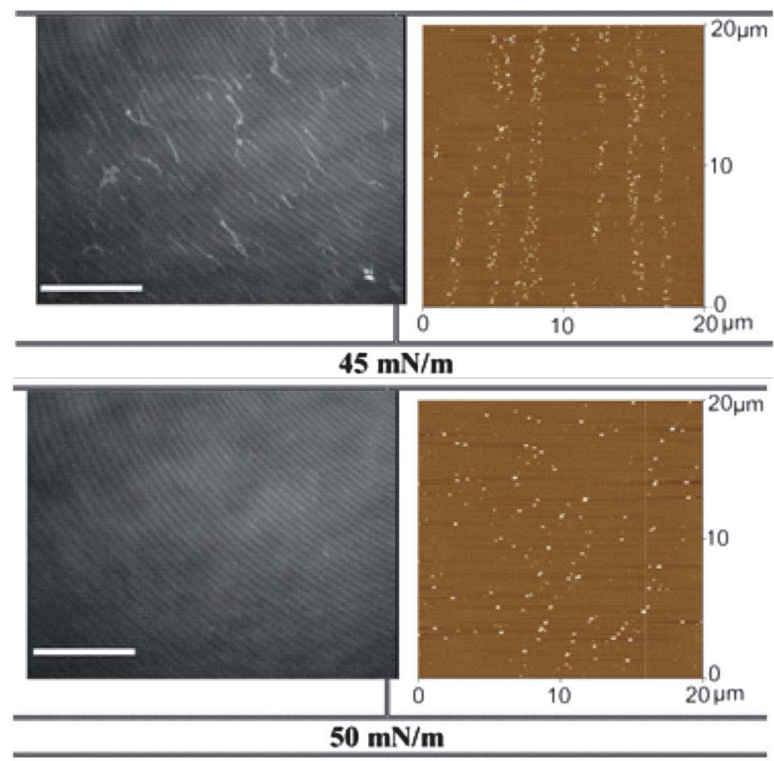

Fig. $9 \mathrm{BAM}$ and AFM images obtained at surface pressures above $25 \mathrm{mN} \mathrm{m}^{-1}$ for the mixed DPPC-Fe $\mathrm{O}_{4} @ \mathrm{MEO}_{2} \mathrm{MA}_{90}-c o-\mathrm{OEGMA}_{10}$ NP layers co-spread on water at $20^{\circ} \mathrm{C}$. For all BAM images, the scale bar is $100 \mu \mathrm{m}$.
BAM images reveal an essentially compact monolayer. However, the higher resolution of the AFM pictures shows that few NPs are still present in the DPPC layer.

The changes in the DPPC monolayer structure produced by the NPs indicate that the polymer shell of the NPs interacts either with the hydrophilic or the hydrophobic part of the phospholipid. The largely observed immiscibility between NPs and DPPC, and the unperturbed characteristic phase transition pressure of DPPC, point to the fact that the NPs have no pronounced interaction with the phospholipid alkyl chains. Thus, the structural changes could be only due to the NPs' influence on the DPPC polar head as discussed above. In this respect, an IRRAS study was conducted for monolayers of pure DPPC, pure NPs and mixed DPPC-NPs. The IR spectra recorded for mixed layers at surface pressures between 10 and $25 \mathrm{mN} \mathrm{m}^{-1}$ revealed a shift of the phosphate asymmetric stretching band to higher wavenumbers (from 1230 to $1237 \mathrm{~cm}^{-1}$ ) when compared to the band recorded for pure DPPC monolayers at the same pressures. This behavior is depicted in Fig. 10A for a mixed DPPC-NPs layer at $20 \mathrm{mN} \mathrm{m}^{-1}$. The blue shift of the maximum intensity of the phosphate asymmetric stretching band indicates that the NPs induce partial dehydration of the DPPC polar head. ${ }^{21,22}$ Interestingly, this behavior is accompanied by a small increase of the dichroic ratio of the same band (Fig. 10C), pointing to a slight conformational change of the DPPC polar head. The same has been observed in the pure DPPC monolayer by compression. This is in line with the GIXD data showing that the area requirement of the DPPC head groups is clearly reduced in the mixed layer. As Fig. 10B and C describe, the NPs' influence on the orientation and hydration of the DPPC polar heads vanishes at surface pressures above $35 \mathrm{mN} \mathrm{m}^{-1}$. Now, the compression has the dominating influence on the structure even if there are still some NPs close to the interface.
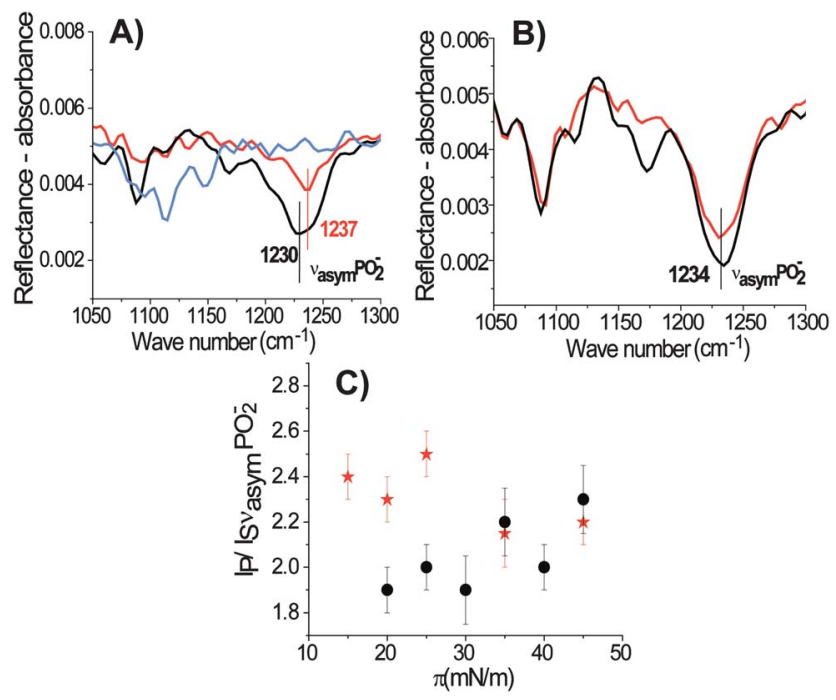

Fig. 10 IRRA spectra of monolayers of pure DPPC (black line), pure $\mathrm{Fe}_{3} \mathrm{O}_{4} @ \mathrm{MEO}_{2} \mathrm{MA}_{90}-\mathrm{co}$-OEGMA 10 NPs (blue line) and mixed NPs/ DPPC (red line) at (A) $20 \mathrm{mN} \mathrm{m}^{-1}$. (B) $45 \mathrm{mN} \mathrm{m}^{-1}$. (C) Dichroic ratio (Ip/Is of the asymmetric $\mathrm{PO}_{2}{ }^{-}$band) of the pure DPPC layer (black dots) and mixed NPs-DPPC (red stars) layer versus the surface pressure of the layers spread on water. 


\section{Conclusions}

Our study is the first one to bring experimental evidence by the use of highly surface sensitive experimental techniques, such as GIXD and IRRAS, for the dehydration effect of the phosphatidylcholine head group induced by a poly(ethylene glycol) based polymer, previously observed by other techniques. ${ }^{23-26}$ It contributes substantially to the understanding of interactions between $\mathrm{Fe}_{3} \mathrm{O}_{4} @ \mathrm{MEO}_{2} \mathrm{MA}_{90}$-co-OEGMA 10 NPs and DPPC molecules in 2D model membranes.

The maximum insertion pressure of the NPs into a DPPC monolayer was determined $\left(25.5 \mathrm{mN} \mathrm{m}^{-1}\right)$ and identified as being controlled by the NPs' surface activity. Following this, BAM experiments showed that the two components are mostly immiscible. Nevertheless, GIXD data revealed that the NPs are able to change the structure of the DPPC monolayers by increasing their molecular in-plane packing due to changed head group hydration and orientation. In this respect, IRRA spectra support the assumed partial dehydration and slight conformational change of the DPPC polar head. Moreover, the study shows that the copolymer is dictating the structural changes in the DPPC monolayers.

So, already in a range of surface pressures $\left(10-25 \mathrm{mN} \mathrm{m}^{-1}\right)$ below the MIP, the NPs force the DPPC molecules to change the monolayer structure to the one found in the pure DPPC Langmuir layers only above $30 \mathrm{mN} \mathrm{m}^{-1}$ (Fig. 11). Additionally, the DPPC-NPs composite is almost incompressible. Interestingly, the induced tighter in-plane packing of DPPC is the key factor which determines the free interfacial area offered to the NPs.

Furthermore, the correlation of the GIXD results with the Langmuir isotherms and the X-ray fluorescence data allowed us to calculate the percentage of the interfacial area occupied by the NPs at different surface pressures in the mixed DPPC-NP layers, including the percentage of squeezed out NPs. Indeed, as previously reported for the pure NP layers, these NPs are trapped at the interface in the mixed DPPC-NP layer up to the critical surface pressure value of $25 \mathrm{mN} \mathrm{m}^{-1}$. This property allows a precise quantification of the interfacial concentration of the NPs. ${ }^{7}$

Above the critical surface pressure, the BAM and AFM data indicate a drastic squeezing out of the NPs, while the GIXD and

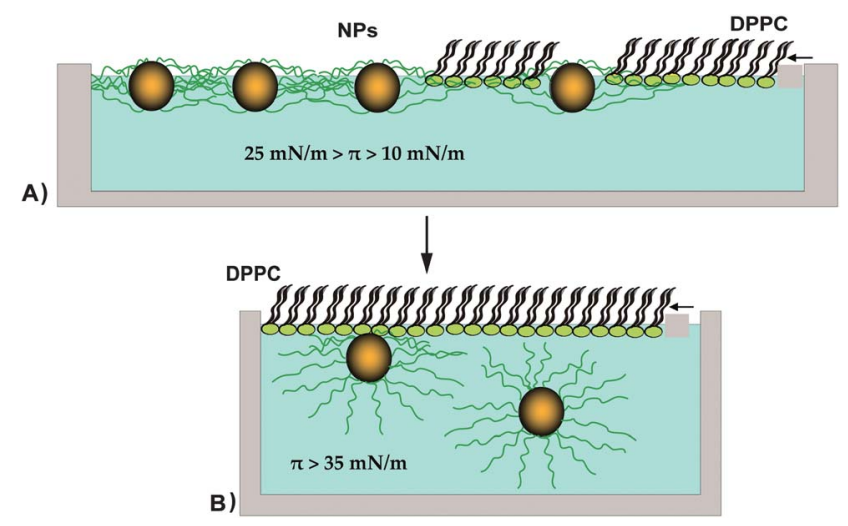

Fig. 11 Schematic representation of the mixed DPPC- $\mathrm{Fe}_{3} \mathrm{O}_{4} @$ $\mathrm{MEO}_{2} \mathrm{MA}_{90}$-co-OEGMA 10 NP layers at different surface pressures.
IRRAS revealed that only above $35 \mathrm{mN} \mathrm{m}^{-1}$ the interfacial layer exhibits the structural characteristics of pure DPPC monolayers.

The finding that the insertion of NPs is controlled by their surface activity could have important practical implications since the NPs' surface activity can be tuned (by changing the molar ratio of the two polymers) and is stimuli dependent (temperature, ionic strength)..$^{5}$ Thus, at the physiological temperature $\left(37^{\circ} \mathrm{C}\right)$, the studied NP system is characterized by a critical surface pressure of $27 \mathrm{mN} \mathrm{m}^{-1} .5$ Considering that the generally accepted value of the lateral surface pressure of a cellular membrane is 30 $\mathrm{mN} \mathrm{m} \mathrm{m}^{-1}$, it is highly probable that these NPs will not have the ability to penetrate undamaged living cell membranes. The only exception could be pressure fluctuation in the membrane allowing their penetration. Nevertheless, the NPs could function as sealing agents of injured cell membranes for which, due to electrical shock or lightning injury, the cell membrane permeability is enhanced due to the loss of the innate packing density. As described for poloxamers, our NPs could seal damaged membranes in the living cells by incorporation, assisting the recovery of the lipid packing, and desorbing thereafter from the restored membrane.

Thus, with this study we showed that the superparamagnetic and stimuli-responsive $\mathrm{Fe}_{3} \mathrm{O}_{4} @ \mathrm{MEO}_{2} \mathrm{MA}_{90}-c o$-OEGMA 10 NPs could be used in the biomedical field not only as magnetic resonance imaging contrast enhancers and cell manipulation agents, but also as cellular membrane sealers.

\section{Acknowledgements}

We are deeply grateful to Dayang Wang and Munish Chanana for the development of the NPs as well as for valuable discussions in the early stage of the project. We thank Mandy Meckelburg for the preparation of the NP colloidal dispersions, Anneliese Heilig for the AFM measurements, HASYLAB at DESY, Hamburg, Germany, and ESRF, Grenoble, France, for beam time and excellent support. This work was supported by the Max Planck Society.

\section{References}

1 W. H. D. Jong and P. J. Borm, Int. J. Nanomed., 2008, 3, 133-149.

2 A. K. Gupta and M. Gupta, Biomaterials, 2005, 26, 3995-4021.

3 M. Chanana, S. Jahn, R. Georgieva, J.-F. Lutz, H. Baumler and D. Wang, Chem. Mater., 2009, 21, 1906-1914.

4 C. Stefaniu, M. Chanana, H. Ahrens, D. Wang, G. Brezesinski and H. Möhwald, Soft Matter, 2011, 7, 4267-4275.

5 C. Stefaniu, M. Chanana, D. Wang, G. Brezesinski and H. Möhwald, J. Phys. Chem. C, 2011, 115, 5478-5484.

6 C. Stefaniu, M. Chanana, D. Wang, D. V. Novikov, G. Brezesinski and H. Möhwald, ChemPhysChem, 2010, 11, 3585-3588.

7 C. Stefaniu, M. Chanana, D. Wang, D. V. Novikov, G. Brezesinski and H. Möhwald, Langmuir, 2011, 27, 1192-1199.

8 D. C. Chang and T. S. Reese, Biophys. J., 1990, 58, 1-12.

9 H. Baskaran, M. Toner, M. L. Yarmush and F. Berthiaume, J. Surg. Res., 2001, 101, 56-61.

10 R. C. Lee, L. P. River, F. S. Pan, L. Ji and R. L. Wollmann, Proc. Natl. Acad. Sci. U. S. A., 1992, 89, 4524-4528.

11 S. A. Maskarinec and K. Y. C. Lee, Langmuir, 2003, 19, 1809-1815.

12 J. R. Charron and R. D. Tilton, J. Phys. Chem., 1996, 100, 3179-3189.

13 J. R. Charron and R. D. Tilton, Langmuir, 1997, 13, 5524-5527.

14 G. Wu, J. Majewski, C. Ege, K. Kjaer, M. J. Weygand and K. Y. C. Lee, Biophys. J., 2005, 89, 3159-3173.

15 K. Wagner and G. Brezesinski, Chem. Phys. Lipids, 2007, 145, 119 127. 
16 K. J. Klopfer and T. K. Vanderlick, J. Colloid Interface Sci., 1996, 182, 220-229.

17 C. W. McConlogue and T. K. Vanderlick, Langmuir, 1997, 13, 7158 7164.

18 E. Amado, A. Kerth, A. Blume and J. Kressler, Langmuir, 2008, 24, 10041-10053.

19 C. Naumann, C. Dietrich, J. R. Lu, R. K. Thomas, A. R. Rennie, J. Penfold and T. M. Bayerl, Langmuir, 1994, 10, 1919-1925.

20 F. Bringezu, B. Dobner and G. Brezesinski, Chem.-Eur. J., 2002, 8, 3203-3210.
21 J. L. R. Arrondo, F. M. Goni and J. M. Macarulla, Biochim. Biophys. Acta, 1984, 794, 165-168.

22 L. M. Crowe, J. H. Crowe and D. Chapman, Arch. Biochem. Biophys., 1985, 236, 289-296.

23 K. Arnold, L. Pratsch and K. Gawrisch, Biochim. Biophys. Acta, 1983, 728, 121-128.

24 J. Y. A. Lehtonen and P. K. J. Kinnunen, Biophys. J., 1995, 68, 525-535.

25 A. Sen and S.-W. Hui, Chem. Phys. Lipids, 1988, 49, 179-184.

26 R. P. Rand and V. A. Parsegian, Biochim. Biophys. Acta, 1989, 988, 351-376. 


\section{Addition and correction}

\section{Note from RSC Publishing}

This article was originally published with incorrect page numbers. This is the corrected, final version.

The Royal Society of Chemistry apologises for these errors and any consequent inconvenience to authors and readers. 